\title{
Optical characterization of ultrahigh diffraction efficiency gratings
}

\author{
A. Bunkowski, O. Burmeister, T. Clausnitzer, E.-B. Kley, A. Tünnermann, \\ K. Danzmann, and R. Schnabel
}

\begin{abstract}
We report on the optical characterization of an ultrahigh diffraction efficiency grating in a first-order Littrow configuration. The apparatus used was an optical cavity built from the grating under investigation and an additional high-reflection mirror. The measurement of the cavity finesse provided precise information about the grating's diffraction efficiency and its optical loss. We measured a finesse of 1580 from which we deduced a diffraction efficiency of $(99.635 \pm 0.016) \%$ and an overall optical loss due to scattering and absorption of just $0.185 \%$. Such high-quality gratings, including the tool used for their characterization, might apply for future gravitational wave detectors. For example, the demonstrated cavity itself presents an all-reflective, low-loss Fabry-Perot resonator that might replace conventional arm cavities in advanced high-power Michelson interferometers. (C) 2006 Optical Society of America

OCIS codes: $\quad 050.1950,230.1360,120.2230$.
\end{abstract}

\section{Introduction}

High-quality optics are key devices in laser interferometric precision measurements. Especially for highpower laser applications with nested cavities, such as gravitational wave detectors, ${ }^{1}$ mirrors with high reflectivity and low overall optical loss are essential. Mirrors with a power reflectance greater than $99.9998 \%$ for a given laser wavelength have been reported. ${ }^{2}$ The overall optical loss consisting of stray light from the surface, transmission, and absorption in the coating was as low as 1.6 parts per million (ppm). ${ }^{2}$

Gratings are traditionally used in applications in which one wants to spatially resolve different optical wavelengths, e.g., in spectrographs or pulse compressors-stretchers for short-pulse laser systems. In these applications, high diffraction efficiency over a range of optical wavelengths is desired. Dielectric reflection gratings with diffraction efficiencies of $96 \%, 97 \%$, and $99 \%$ have been reported. ${ }^{3-5}$ However,

A. Bunkowski (alexander.bunkowski@aei.mpg.de), O. Burmeister, K. Danzmann, and R. Schnabel are with the MaxPlanck-Institut für Gravitationsphysik (Albert-Einstein-Institut) and Universität Hannover, Callinstrasse 38, 30167 Hannover, Germany. T. Clausnitzer, E.-B. Kley, and A. Tünnermann are with the Institut für Angewandte Physik, Friedrich-SchillerUniversität Jena, Max-Wien-Platz 1, 07743 Jena, Germany.

Received 6 December 2005; accepted 3 March 2006; posted 17 March 2006 (Doc. ID 66481).

0003-6935/06/235795-05\$15.00/0

(C) 2006 Optical Society of America the measurement techniques used in those applications allowed for only a rough estimation of the diffraction efficiency, and no error bars for the values were given. Diffraction gratings may also be used in advanced high-power laser interferometers, ${ }^{6,7}$ where they allow for the all-reflective realization of beam splitters and cavity couplers, and therefore may help reduce thermal effects in the substrate, such as thermal lensing ${ }^{8}$ and thermorefractive noise. ${ }^{9}$ In interferometric applications, monochromatic laser light is used, and the wavelength dispersive property of the gratings is not essential. The point of interest lies in the number and the properties of the reflective diffraction ports and their couplings that determine the interference between input beams. Two different allreflective resonator concepts have been demonstrated to date. High-efficiency gratings in first-order Littrow configuration form cavity couplers with two ports analogous to partially transmitting mirrors. ${ }^{7}$ Lowefficiency gratings in second-order Littrow configuration can be used as low-loss couplers with three ports. ${ }^{10}$ Analogous to conventional mirrors, however, optical loss in terms of scattering or absorption has to be minimized to gain maximum laser power buildup and measurement sensitivity. The question therefore arises as to whether high-efficiency gratings with highly corrugated surfaces will ever be able to fulfill the strict low scattering loss requirements.

In this paper we report on the optical characterization of a high-efficiency grating in view of applications in interferometry. The grating was used in a 


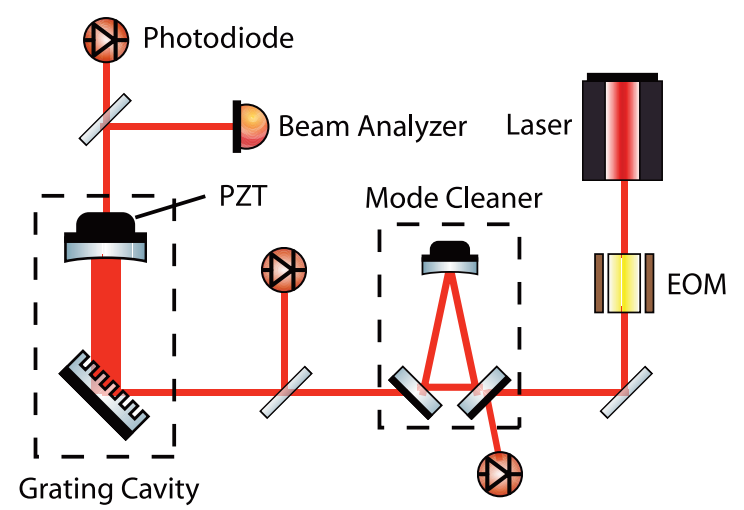

Fig. 1. (Color online) Experimental setup; EOM, electro-optical modulator; PZT, cavity mirror with a piezoelectric transducer for length control.

first-order Littrow configuration to couple laser light into a Fabry-Perot cavity with a measured finesse of $1580 \pm 60$. This experiment allowed for the accurate measurement of both the grating's loss and the diffraction efficiency. The latter was determined to be $(99.635 \pm 0.016) \%$. To our knowledge this is the highest and most accurately determined value reported in the literature.

The grating device was designed for a laser wavelength of $1064 \mathrm{~nm}$ and a Littrow angle of approximately $30^{\circ}$. The grating structure had rectangular grooves with a period of $1060 \mathrm{~nm}$. For fabrication we used electron-beam direct writing (electron-beam writer LION LV1, Leica Microsystems GmbH) and reactive ion-beam etching into the top layer of a highly reflective dielectric multilayer stack. The stack consisted of 36 alternating layers of $195 \mathrm{~nm}$ $\mathrm{SiO}_{2}$ and $136 \mathrm{~nm} \mathrm{Ta}_{2} \mathrm{O}_{5}$ placed on a fused-silica substrate with a surface flatness of $\lambda / 10$. For the theoretical optimization of the grating we used the rigorous coupled-wave analysis. ${ }^{11}$ To ensure good reproducibility and homogeneity over the whole grating area, an important design concern was a large groove parameter tolerance of diffraction efficiency. By using $\mathrm{SiO}_{2}$ with a thickness of $1.12 \mu \mathrm{m}$ as the top layer of the dielectric stack the theoretical design exhibited a diffraction efficiency of more than $99 \%$ for groove depths between 700 and $850 \mathrm{~nm}$ and groove widths between 530 and $760 \mathrm{~nm}$.

\section{Experimental Procedure}

A schematic of our experiment is seen in Fig. 1. The light source used was a $1.2 \mathrm{~W}$ diode-pumped Nd:YAG laser at $1064 \mathrm{~nm}$ (Model Mephisto, Innolight $\mathrm{GmbH}$ ). Before the $s$-polarized laser beam was sent into the grating cavity it was spatially filtered with a triangular ring cavity (mode cleaner). ${ }^{12}$ The highly reflective end mirror of the grating cavity was mounted on a piezoelectric transducer (PZT) that was used either to scan or to actively control the cavity length. The error signal for the electronic servo loop was obtained from the cavity transmission demodulated at the phase modulation frequency introduced by the electro-optical modulator (EOM).

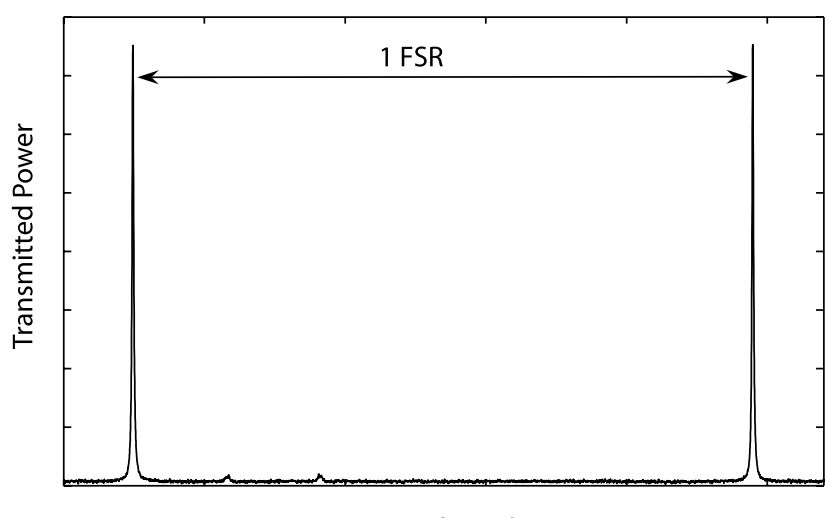

\section{Cavity Length}

Fig. 2. Transmitted power of the cavity. Photodiode signal behind the grating cavity as the cavity is linearly scanned over one free spectral range (FSR).

In a first-order Littrow configuration, only two diffraction orders exist, and the grating (subscript 1) is characterized by the zeroth and first-order amplitude diffraction efficiencies $r_{1}$ and $\eta_{1}$, respectively, as well as the loss amplitude $l_{1}$. Similarly, the cavity end mirror (subscript 2) is described by $r_{2}, t_{2}$, and $l_{2}$. Energy conservation implies

$$
\begin{gathered}
r_{1}^{2}+\eta_{1}^{2}+l_{1}^{2}=1, \\
r_{2}^{2}+t_{2}^{2}+l_{2}^{2}=1 .
\end{gathered}
$$

Figure 2 shows the transmission spectrum of the cavity as the PZT is linearly scanned over one free spectral range of the cavity. In addition to the peaks of the fundamental mode of the cavity there are only two smaller peaks from higher-order modes visible, indicating a good matching of laser beam and cavity mode.

A method to obtain a precise value for a mirror reflectance close to unity is a measurement of the finesse $F$ of a cavity consisting of a mirror with a known reflectance and the one in question. For the first time, to the best of our knowledge, this method is applied to characterize a high-efficiency grating. If losses due to absorption in the space between the mirrors (which would appear additionally to $l_{1}$ and $l_{2}$ ) are neglected, the finesse $F$ of a two-mirror FabryPerot resonator depends on the reflectance of the two end mirrors only. In our case one of the end mirrors is a grating, and the finesse can be approximated by

$$
F=\pi\left(\eta_{1} r_{2}\right)^{1 / 2} /\left(1-\eta_{1} r_{2}\right) .
$$

For a cavity of length $L$ its free spectral range is given by $f_{\mathrm{FSR}}=c / 2 L$, where $c$ is the speed of light. The ratio of $f_{\mathrm{FSR}}$ to the FWHM $f_{\mathrm{FWHM}}$ of the Airy transmission spectrum peaks determines the finesse:

$$
F=f_{\mathrm{FSR}} / f_{\mathrm{FWHM}} .
$$

The length of the cavity was measured to $L=$ 


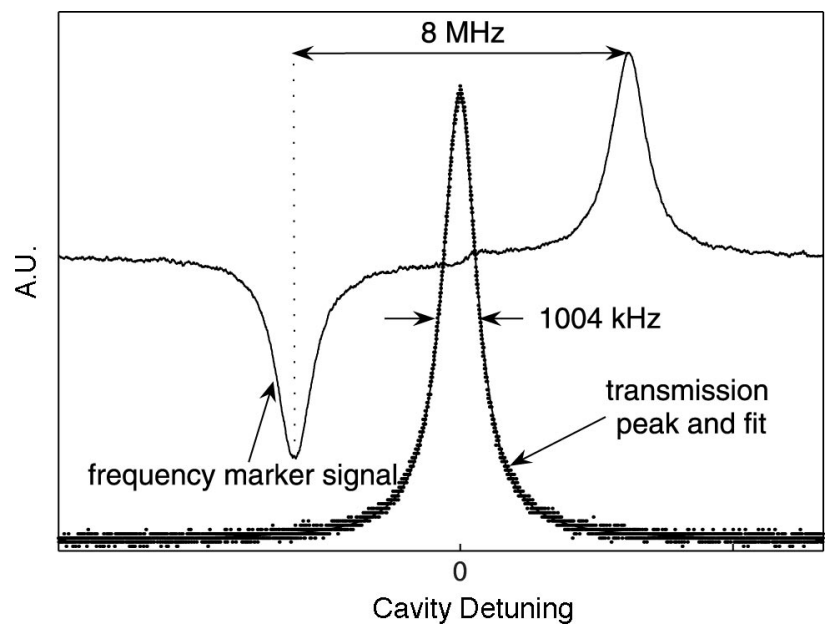

Fig. 3. Scan over one cavity transmission peak. The $x$ axis was calibrated with $\pm 4 \mathrm{MHz}$ marker signals.

$(94 \pm 1) \mathrm{mm}$, resulting in $f_{\mathrm{FSR}} \approx 1.6 \mathrm{GHz}$. The cavity linewidth was measured by utilizing frequency marker signals. The laser light was phase modulated at $f_{\text {mod }}=4 \mathrm{MHz}$ by using an EOM. The ac output of the photodiode in front of the grating cavity was then demodulated at $f_{\text {mod. }}$. For the correct demodulation phase this signal shows a minimum and a maximum at exactly $\pm f_{\bmod }$ and can be used to calibrate the $x$ axis in Fig. 3. The figure shows a typical measured dc signal of the photodiode behind the cavity as well as the marker signals at $f_{\text {marker }}= \pm(4 \pm 0.04) \mathrm{MHz}$ while the cavity was linearly scanned with a $1 \mathrm{kHz}$ repetition rate. The uncertainty in the position of the marker signal is due to an error in the demodulation phase. A fit of the transmission signal to the wellknown Airy function of cavities permitted the calculation of the width of the transmission peak. Due to nonlinearities in the PZT and acoustic vibrations, there is a statistical variation of the linewidth of the peak. We averaged over 75 measurements by using different operating points of the PZT and we could reduce the statistical error in the peak width to $\pm 3.5 \%$.

With Eq. (4) we could calculate the finesse of the cavity $F=1580 \pm 60$. The cavity end mirror was superpolished and coated by Research ElectroOptics, Inc., and specified to have values of $t_{2}{ }^{2}=300 \pm 30 \mathrm{ppm}$ and $l_{2}{ }^{2}<30 \mathrm{ppm}$. From these specifications we estimated the mirror's reflectivity to $r_{2}{ }^{2}=(99.9685 \pm 0.0034) \%$. With Eqs. (2) and (3) we obtained $\eta_{1}{ }^{2}=(99.635 \pm 0.016) \%$ for the grating's first-order diffraction efficiency. The error in $\eta_{1}{ }^{2}$ results from an error propagation of each known uncertainty of the quantities $L, f_{\text {marker }}$, fitted peak width, and $r_{2}{ }^{2}$ as shown in Table 1 . The specular reflection of the grating was measured independently with a calibrated powermeter to be $r_{1}{ }^{2}=(0.18 \pm 0.009) \%$. Hence we calculated the overall loss of the grating according to Eq. (1) to be $l_{1}{ }^{2}=(0.185 \pm 0.025) \%$. We emphasize that this loss contained all contributions from scattering, absorption, transmission, and
Table 1. Error Propagation

\begin{tabular}{llc}
\hline \multicolumn{1}{c}{ Quantity } & Error & $\begin{array}{c}\text { Projected Error } \\
\text { for } \eta_{1}{ }^{2}(\mathrm{ppm})\end{array}$ \\
\hline $\mathrm{L}$ & $\pm 1 \mathrm{~mm}$ & \pm 48 \\
$f_{\text {marker }}$ & $\pm 40 \mathrm{kHz}$ & \pm 43 \\
Peak width & $\pm 3.5 \%$ & \pm 143 \\
$r_{2}{ }^{2}$ & $\pm 34 \mathrm{ppm}$ & \pm 34 \\
Total rms error & & \pm 160 \\
$\quad$ expected & & \\
\hline
\end{tabular}

higher diffraction orders. To the best of our knowledge, this result presents the lowest and most accurately determined grating loss reported in the literature. The previous results were those by Perry et al. ${ }^{3}$ and Hehl et al. ${ }^{4}$ who reported a $1.5 \%$ and a 1\%-2\% loss, respectively. Destouches et al. ${ }^{5}$ did not comment on the loss.

In addition to the grating's loss we also investigated its influence on the laser beam's spatial profile. Again a cavity in a first-order Littrow configuration was set up with a cavity mode waist on the grating's surface now using an end mirror with a power reflectivity of ${r_{2}}^{2}=99 \%$ to reduce the finesse value and to increase transmission. The cavity length was controlled by use of a Pound-Drever-Hall locking scheme with a phase modulation sideband frequency of $4 \mathrm{MHz}$. The beam profile for the horizontal and vertical directions was measured after the cavity by using a seven-blade tomographic profiler (SuperBeamAlyzer, Melles Griot) fitted with a Gaussian model, as shown in Fig. 4. The sum of the absolute differences between the value of every measured point and the fitted function divided by the sum of the values of all fitted points is a measure of how much beam power can be represented by a Gaussian function. For both directions we
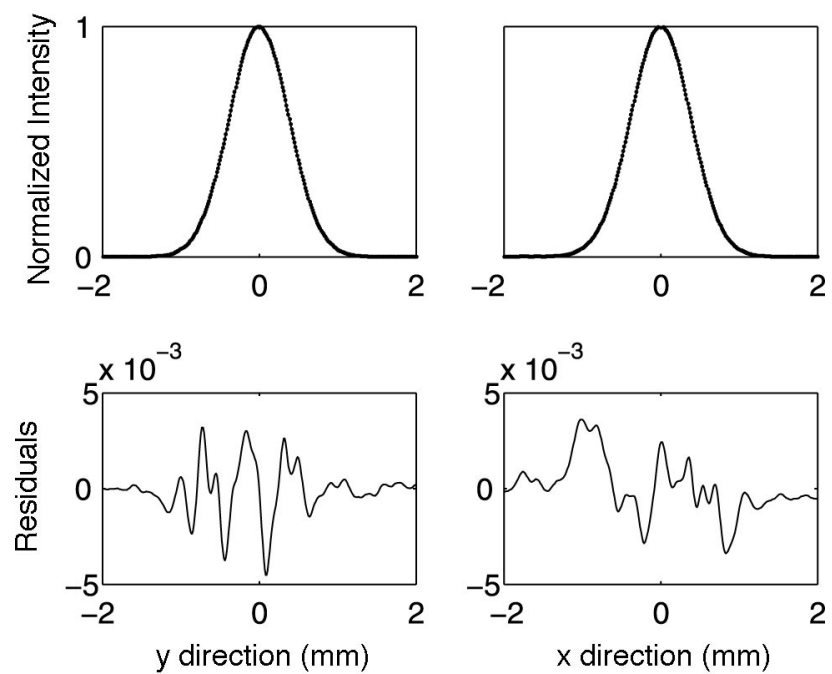

Fig. 4. Spatial beam profile of the laser beam after the cavity for horizontal (perpendicular to the grating lines) and vertical (parallel to the grating lines) directions. Top, measured points (dots) and best Gaussian fit (solid curve); bottom, residuals between measurement and fit. 
obtained values of greater than $99 \%$. For this experiment, the mode cleaner had been taken out, which allowed us to observe a mode-cleaning effect from the grating cavity. We characterized the laser beam behind the EOM by using the same apparatus and got spatial profiles that were described by a Gaussian function by only $98 \%$.

\section{Conclusion}

We presented a detailed characterization of diffraction efficiency and overall loss of a grating in a first-order Littrow mount. The grating's diffraction efficiency showed an outstanding high value that permitted the construction of a high-finesse cavity as a characterizing tool. The value of the finesse was limited by the first-order diffraction efficiency. This is in contrast to Ref. 10 in which a low diffraction efficiency grating was characterized with a high-finesse cavity and the limit for the finesse was given by the specular reflectivity of the grating. Our approach is a valuable diagnostic tool to improve future techniques of grating fabrication since all types of loss are simultaneously detected. We expect that with improved technology high grating efficiencies with simultaneously low loss are possible and that they will even fulfill the strict requirements of future interferometers, such as those for gravitational wave detection.

This work was supported by the Deutsche Forschungsgemeinschaft within the Sonderforschungsbereich TR7.

\section{References}

1. B. Abbott, R. Abbott, R. Adhikari, A. Ageev, B. Allen, R. Amin, S. B. Anderson, W. Anderson, M. Araya, H. Armandula, F. Asiri, P. Aufmuth, C. Aulbert, S. Babak, R. Balasubramanian, S. Ballmer, B. C. Barish, D. Barker, C. Barker-Patton, C. M. Barnes, B. Barr, M. A. Barton, K. Bayer, R. Beausoleil, K. Belczynski, R. Bennett, S. J. Berukoff, J. Betzwieser, B. Bhawal, I. A. Bilenko, G. Billingsley, E. Black, K. Blackburn, B. Bland-Weaver, B. Bochner, L. Bogue, R. Bork, S. Bose, P. R. Brady, V. B. Bragensky, J. E. Brau, D. A. Brown, S. Brozek, A. Bullington, A. Buonano, R. Burgess, D. Busby, W. E. Butler, R. L. Byer, L. Cadonati, G. Cagnoli, J. B. Camp, C. A. Cantley, L. Cardenas, K. Carter, M. M. Casey, J. Castiglione, A. Chandler, J. Chapsky, P. Charlton, S. Chatterji, Y. Chen, V. Chickarmane, D. Chin, N. Christensen, D. Churches, C. Colacino, R. Coldwell, M. Coles, D. Cook, T. Corbitt, D. Coyne, J. D. E. Creighton, T. D. Creighton, D. R. M. Crooks, P. Csatorday, B. J. Cusack, C. Cutler, E. D’Ambrosio, K. Danzmann, R. Davies, E. Daw, D. DeBra, T. Delker, R. DeSalvo, S. Dhurandar, M. Diaz, H. Ding, R. W. P. Drever, R. J. Dupuis, C. Ebeling, J. Edlund, P. Ehrens, E. J. Elliffe, T. Etzel, M. Evans, T. Evans, C. Fallnich, D. Farnham, M. M. Fejer, M. Fine, L. S. Finn, E. Flanagan, A. Freise, R. Frey, P. Fritschel, V. Frolov, M. Fyffe, K. S. Ganezer, J. A. Giaime, A. Gillespie, K. Goda, G. Gonzalez, S. Gossler, P. Grandclement, A. Grant, C. Gray, A. M. Gretarsson, D. Grimmett, H. Grote, S. Grunewald, M. Guenther, E. Gustafson, R. Gustafson, W. O. Hamilton, M. Hammond, J. Hanson, C. Hardham, G. Harry, A. Hartunian, J. Heefner, Y. Hefetz, G. Heinzel, I. S. Heng, M. Hennessy, N. Hepler, A. Heptonstall, M. Heurs, M. Hewitson, N. Hindman, P. Hoang, J. Hough, M. Hrynevych, W. Hua, R. Ingley, M. Ito, Y. Itoh, A. Ivanov, O. Jennrich, W. W. Johnson, W. Johnston, L. Jones, D.
Jungwirth, V. Kalogera, E. Katsavounidis, K. Kawabe, S. Kawamura, W. Kells, J. Kern, A. Khan, S. Killbourn, C. J. Killow, C. Kim, C. King, P. King, S. Klimenko, P. Kloevekorn, S. Koranda, K. Kotter, J. Kovalik, D. Kozak, B. Krishnan, M. Landry, J. Langdale, B. Lantz, R. Lawrence, A. Lazzarini, M. Lei, V. Leonhardt, I. Leonor, K. Libbrecht, P. Lindquist, S. Liu, J. Logan, M. Lormand, M. Lubinski, H. Luck, T. T. Lyons, B. Machenschalk, M. MacInnis, M. Mageswaran, K. Mailand, W. Majid, M. Malec, F. Mann, A. Marin, S. Marka, E. Maros, J. Mason, K. Mason, O. Matherny, L. Matone, N. Mavalvala, R. McCarthy, D. E. McClelland, M. McHugh, P. McNamara, G. Mendell, S. Meshkov, C. Messenger, V. P. Metrofanov, G. Mitselmakher, R. Mittleman, O. Miyakawa, S. Miyoki, S. Mohanty, G. Moreno, K. Mossavi, B. Mours, G. Mueller, S. Mukherjee, J. Myers, S. Nagano, T. Nash, H. Naundorf, R. Nayak, G. Newton, F. Nocera, P. Nutzman, T. Olson, B. O'Reilly, D. J. Ottaway, A. Ottewill, D. Ouimette, H. Overmier, B. J. Owen, M. A. Papa, C. Parameswariah, V. Parameswariah, M. Pedraza, S. Penn, M. Pitkin, M. Plissi, M. Pratt, V. Quetschke, F. Raab, H. Radkins, R. Rahkola, M. Rakhmanov, S. R. Rao, D. Redding, M. W. Regehr, T. Regimbau, K. T. Reilly, K. Reithmaier, D. H. Reitze, S. Richman, R. Riesen, K. Riles, A. Rizzi, D. I. Robertson, N. A. Robertson, L. Robison, S. Roddy, J. Rollins, J. D. Romano, J. Romie, H. Rong, D. Rose, E. Rotthoff, S. Rowan, A. Rudiger, P. Russell, K. Ryan, I. Salzman, G. H. Sanders, V. Sannibale, B. Sathyaprakash, P. R. Saulson, R. Savage, A. Sazonov, R. Schilling, K. Schlaufman, V. Schmidt, R. Schofield, M. Schrempel, B. F. Schutz, P. Schwinberg, S. M. Scott, A. C. Searle, B. Sears, S. Seel, A. S. Sengupta, C. A. Shapiro, P. Shawhan, D. H. Shoemaker, Q. L. Shu, A. Sibley, X. Siemens, L. Sievers, D. Sigg, A. M. Sintes, K. Skeldon, J. R. Smith, M. Smith, M. R. Smith, P. Sneddon, R. Spero, G. Stapfer, K. A. Strain, D. Strom, A. Stuver, T. Summerscales, M. C. Sumner, P. J. Sutton, J. Sylvestre, A. Takamori, D. B. Tanner, H. Tariq, I. Taylor, R. Taylor, K. S. Thorne, M. Tibbits, S. Tilav, M. Tinto, K. V. Tokmakov, C. Torres, C. Torrie, S. Traeger, G. Traylor, W. Tyler, D. Ugolini, M. Vallisneri, M. van Putten, S. Vass, A. Vecchio, C. Vorvick, S. P. Vyatchanin, L. Wallace, H. Walther, H. Ward, B. Ware, K. Watts, D. Webber, A. Weidner, U. Weiland, A. Weinstein, R. Weiss, H. Welling, L. Wen, S. Wen, J. T. Whelan, S. E. Whitcomb, B. F. Whiting, P. A. Willems, P. R. Williams, R. Williams, B. Willke, A. Wilson, B. J. Winjum, W. Winkler, S. Wise, A. G. Wiseman, G. Woan, R. Wooley, J. Worden, I. Yakushin, H. Yamamoto, S. Yoshida, I. Zawischa, L. Zhang, N. Zotov, M. Zucker, J. Zweizig, and the LIGO Scientific Collaboration, "Detector description and performance for the first coincidence observations between LIGO and GEO," Nucl. Instrum. Methods Phys. Res. A 517, 154-179 (2004).

2. G. Rempe, R. J. Thompson, H. J. Kimble, and R. Lalezari, "Measurement of ultralow losses in an optical interferometer," Opt. Lett. 17, 363-365 (1992).

3. M. D. Perry, R. D. Boyd, J. A. Britten, D. Decker, B. W. Shore, C. Shannon, and E. Shults, "High-efficiency multilayer dielectric diffraction gratings," Opt. Lett. 20, 940-943 (1995).

4. K. Hehl, J. Bischoff, U. Mohaupt, M. Palme, and B. Schnabel, "High-efficiency dielectric reflection gratings: design, fabrication, and analysis," Appl. Opt. 38, 6257-6271 (1999).

5. N. Destouches, A. V. Tishchenko, J. C. Pommier, S. Reynaud, O. Parriaux, S. Tonchev, and M. Abdou Ahmed, "99\% efficiency measured in the -1st order of a resonant grating," Opt. Express 13, 3230-3235 (2005).

6. R. W. P. Drever, "Concepts for extending the ultimate sensitivity of interferometric gravitational wave detectors using non-transmissive optics with diffractive or holographic coupling," in Proceedings of the Seventh Marcel Grossman Meeting 
on General Relativity, M. Keiser and R. T. Jantzen, eds. (World Scientific, 1995) pp. 1401-1406.

7. K.-X. Sun and R. L. Byer, "All-reflective Michelson, Sagnac, and Fabry-Perot interferometers based on grating beam splitters," Opt. Lett. 23, 567-569 (1997).

8. W. Winkler, K. Danzmann, A. Rüdiger, and R. Schilling, "Heating by optical absorption and the performance of interferometric gravitational-wave detectors," Phys. Rev. A. 44, 7022-7036 (1991)

9. V. B. Braginsky, M. L. Gorodetsky, and S. P. Vyatchanin, "Thermo-refractive noise in gravitational wave antennae," Phys. Lett. A 271, 303-307 (2000).
10. A. Bunkowksi, O. Burmeister, P. Beyersdorf, K. Danzmann, R. Schnabel, T. Clausnitzer, E.-B. Kley, and A. Tünnermann, "Low-loss grating for coupling to a high-finesse cavity," Opt. Lett. 29, 2342-2344 (2004).

11. M. G. Moharam and T. K. Gaylord, "Diffraction analysis of dielectric surface-relief gratings," J. Opt. Soc. Am. 72, 13851392 (1982).

12. B. Willke, N. Uehara, E. K. Gustafson, R. L. Byer, P. J. King, S. U. Seel, and R. L. Savage, Jr., "Spatial and temporal filtering of a $10 \mathrm{~W}$ Nd:YAG laser with a Fabry-Perot ring-cavity premode cleaner," Opt. Lett. 23, 1704-1706 (1998). 